\title{
Perceptions of trophectoderm as a sentinel for embryo selection
}

\author{
David F. Albertini
}

Published online: 11 November 2014

(C) Springer Science+Business Media New York 2014

The archives of mammalian embryology are punctuated by landmark studies covering a rich tradition of technological bravado that spans 75 years. The most primordial perceptions of preimplantation embryo morphology were recorded at the Carnegie Institute for Embryology in Baltimore by the likes of Corner, Streeter, Hauser, Hartmann and others in the 1930s and 1940s in prosimian forbears and set the stage for the meticulous dissections and consequent descriptions of we now refer to as the "egg hunts" from the Hertig and Rock era. Shedding light on the earliest stages of human development was hardly a matter of academic pursuit! In fact for the history buffs among our readership, even a cursory examination of Hertig's classic "The Human Trophoblast" published in 1966 will prompt a smile or two from his description of the use of a car battery and headlight to obtain enough transillumination in the dissecting microscope to permit identification of the 20ish early-stage embryos he extricated while they were in transit through the Fallopian tubes.

Around the same time that Hertig's treatise was published in the mid 1960s, Professor Mulnard in Brussels was recording the development of mouse embryos by time lapse microscopy. These prescient studies set a benchmark in the field for several reasons. For one, the fact that development of a mouse embryo could be sustained for 5 days after fertilization in vivo implied the existence of inherent resilience ex vivo in a simple culture medium as long as physiological temperatures were maintained. But the most insightful findings Mulnard introduced to the field had to do with the processes of compaction,

Capsule The quest continues for reliable biomarkers in human blastocysts that will predict the chances of successful pregnancy. In this issue, recent efforts ranging from cost effective molecular genetics to high resolution imaging desperately seeking the holy grail for embryo selection sought by practitioners of human ARTs.

D. F. Albertini $(\bowtie)$

University of Kansas Medical Center, Kansas, KS, USA

e-mail: dalbertini@kumc.edu cavitation, and blastocyst expansion, revealed for the first time as dynamic and sequential steps for each of the embryos he was able to capture complete development. Finally and most dramatically, for those who have had the chance to see his original movies, the ability of the blastocyst to collapse and expand repeatedly was to the naïve observer a most striking and thought provoking behavior that begged explanation and a raison d'être. Fast-forward 50 years with the ever-expanding use of time lapse microscopy, we take as commonplace such behaviors in the thousands of human embryos witnessed throughout the world on a daily basis.

Has the availability of this expansive database of preimplantation embryo dynamism afforded the practical wherewithal for improving embryo selection and pregnancy rates? Maybe. Time will tell whether the machine-vision recording and algorithm laced feature extraction emerging in the field of time lapse miscroscopy will unveil the deepest secrets of embryos destined to produce a live birth, or not. Alone, or in conjunction with time lapse, we continue to see refinements in the seasoned art of critical observation and molecular genetic approaches that share in the spirit of optimizing embryo selection for patients and practitioners alike. And this month, JARG brings to our readership a glimmer of hope with several papers aimed exactly at satisfying such aspirations.

Within a more traditional realm, observational studies have for years attempted to tease apart the morphological subtleties underlying a given embryo's developmental fitness, and the paper by Chen and colleagues takes us one important step closer to this goal (Trophectoderm morphology predicts outcomes of pregnancy in vitrified-warmed single-blastocyst transfer cycle in a Chinese population, DOI 10.1007/ s10815-014-0317). The group from the Nanjing Medical University set about discriminating between ICM, trophectoderm (TM), and blastocyst expansion properties in cohorts of IVF or ICSI embryos derived in all cases from vitrified blastocyst SET cycles. When clinical pregnancy, live 
birth, and miscarriage rates were used as outcome measures, only grading of TM (compared to ICM nor blastocyst expansion) proved to have predictive value. More than anything else, studies of this kind require more robust data sets before firm conclusions can be reached and for those supporters of extended Day 5 culture and SET after blast vitrification some benefit is on the horizon.

From the molecular genetic angle of TE evaluation comes a paper from one of the leading groups in the area of PGS (Scott et al., Trophectoderm DNA fingerprinting by quantitative real-time PCR successfully distinguishes sibling human embryos, DOI 10.1007/s10815-014-0315). While the resilience of biopsied human embryos at either compaction or blastocyst stages for purposes of PGS continues to be discussed and tested given the necessity for improved embryo selection strategies, the costs of whole genome amplification approaches forecast for the immediate future remains an economic deterrent. Thus, exploring more cost-effective technologies in the world of PGS, especially as applied to adoption of TE biopsies, is warranted at this time. Towards this end, the study from RMA New Jersey asks whether more cost-effective alternatives can be brought to bear on the problem of high-resolution discrimination between sibling embryos where tracking outcomes after multiple embryo transfer would be desirable and informative. In this case, proof of principle is provided for qPCR-based DNA fingerprinting technology in TE biopsies that permits allelic differentiation with 40 SNPs. The predicted power and relatively low cost for such a procedure is good news for a field in search of a "home" for the TE samples as they are accumulated at more centers hopeful of establishing a defining sentinel for the best of the best to transfer.

We hope our readership will also take to heart the wide offerings of this issue of JARG, and we look forward to sharing with you next month a repeat performance of the "best of reproductive medicine" from 2014. 